\title{
Spontaneous umbilical cord hematoma as a clinical and forensic medical problem in case of suspected obstetrician's medical malpractice
}

\author{
Rafał Skowronek ${ }^{1}$, Mateusz Zamłyński², Maciej Kajor ${ }^{3}$, \\ Jacek Zamłyński², Małgorzata Chowaniec ${ }^{4}$
}

${ }^{1}$ Department of Forensic Medicine and Forensic Toxicology, School of Medicine in Katowice, Medical University of Silesia in Katowice, Poland

${ }^{2}$ Department of Gynecology, Obstetrics and Gynecologic Oncology in Bytom,

School of Medicine With the Division of Dentistry in Zabrze, Medical University of Silesia in Katowice, Poland

${ }^{3}$ Chair and Department of Pathomorphology and Molecular Diagnostics,

School of Medicine in Katowice, Medical University of Silesia in Katowice, Poland

${ }^{4}$ Chair and Department of Human Anatomy, School of Medicine in Katowice, Medical University of Silesia in Katowice, Poland

We present a case from our forensic medical expertise practice of a spontaneous umbilical cord hematoma finding during the operational completion of a 41-week pregnancy due to an intrauterine fetal distress.

Spontaneous umbilical cord hematoma is rarely the cause of perinatal fetal death. It occurs in 1:5500 pregnancies. The most common risk factors are: infections, morphological anomalies, changes in the walls of vessels or prolapse and twisting of the umbilical cord. In many cases, the cause of hematoma within the umbilical cord remains unknown. Most often, this pathology occurs at the end of pregnancy in the perinatal period. The hematoma is most frequently located in the fetal end of umbilical cord. It can lead to the compression of umbilical vessels resulting in impaired blood flow, acute hypoxia, and in extremely unfavorable cases - fetal death. The diagnosis is most often made postnatally during the assessment of the umbilical cord and placenta.

An unigravida aged 25 was admitted to the obstetric pathology department 10 days after the expected date of delivery. The course of pregnancy was normal. On admission the fetus was in occipito longintudinal position, fetal heart sounds were rhythmical, $140 \mathrm{bpm}$. The patient was qualified for a preinduction of delivery by a Foley catheter. Due to incorrect CTG records (including fetal bradycardia up to $80 \mathrm{bpm}$ ), the decision was made to end the pregnancy by emergency cesarean section. A neonate, weighing $3950 \mathrm{~g}, 60 \mathrm{~cm}$ long, was born in a severe condition (1 point on the Apgar scale). Amniotic fluid was clean. The length of the umbilical cord was $39 \mathrm{~cm}$. Within it, closer to the fetus, a dark-violet $15-\mathrm{cm}$ section was found. After the delivery the complete placenta was sent to the pathomorphology department. In addition to the presence of an extravascular hematoma of unknown aetiology (Fig. 1), the studies showed no structural abnormalities in the structure of the umbilical cord within its stroma and wall (Fig. 2). Also within the placenta (Fig. 3) and fetal membranes (Fig. 4), apart from the changes typical to the time of pregnancy, no morphologically available pathology was found.

During the forensic medical assessment of this case, ordered by the court, one of the most significant problems was the inability to clearly determine the causes and timing of the above described umbilical cord hematoma. Therefore, in similar

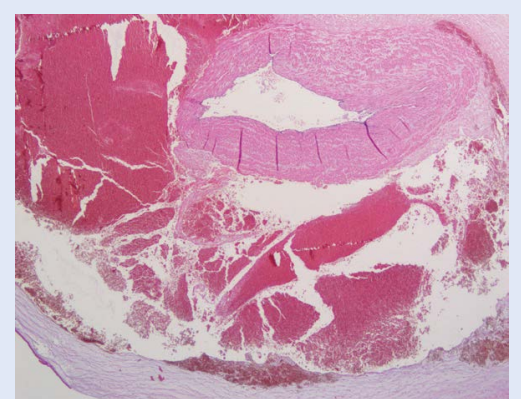

Figure 1. Cross-section of the umbilical cord at the site of extravascular hematoma. Magnification 20x

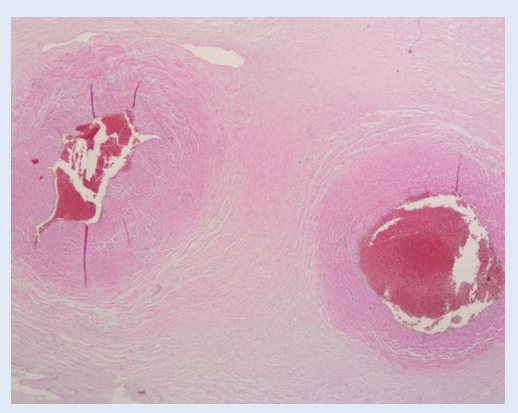

Figure 2. Cross-section of the umbilical cord in the unchanged place. Magnification 20x

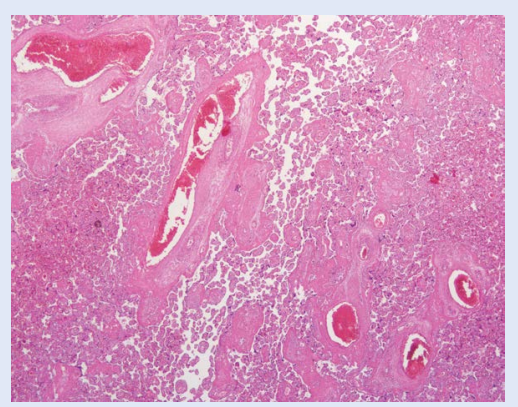

Figure 3. Placenta. Magnification 20x 
cases with the image suggesting the possibility of the presence of a spontaneous umbilical cord hematoma, an accurate, intraoperative morphological description of the lesion, and full post-mortem and histopathological examination of placenta and fetus (in lethal cases) should be performed.

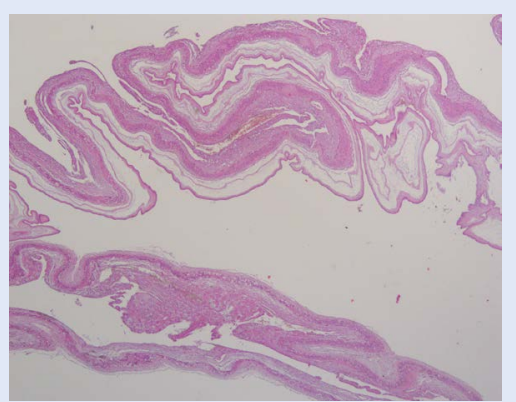

Figure 4. Fetal membranes. Magnification 20x 